\title{
Accuracy of robot-guided versus freehand fluoroscopy-assisted pedicle screw insertion in thoracolumbar spinal surgery
}

\author{
Granit Molliqaj, MD, ${ }^{1}$ Bawarjan Schatlo, MD, ${ }^{2}$ Awad Alaid, MD, ${ }^{2}$ Volodymyr Solomiichuk, MD, ${ }^{2}$ \\ Veit Rohde, MD, ${ }^{2}$ Karl Schaller, MD, ${ }^{1}$ and Enrico Tessitore, MD ${ }^{1}$ \\ 1Department of Neurosurgery, Geneva University Hospitals, Faculty of Medicine, University of Geneva, Switzerland; and \\ 2Department of Neurosurgery, Göttingen University Hospital, Georg-August-University Göttingen, Germany
}

\begin{abstract}
OBJECTIVE The quest to improve the safety and accuracy and decrease the invasiveness of pedicle screw placement in spine surgery has led to a markedly increased interest in robotic technology. The SpineAssist from Mazor is one of the most widely distributed robotic systems. The aim of this study was to compare the accuracy of robot-guided and conventional freehand fluoroscopy-guided pedicle screw placement in thoracolumbar surgery.

METHODS This study is a retrospective series of 169 patients (83 women [49\%]) who underwent placement of pedicle screw instrumentation from 2007 to 2015 in 2 reference centers. Pathological entities included degenerative disorders, tumors, and traumatic cases. In the robot-assisted cohort (98 patients, 439 screws), pedicle screws were inserted with robotic assistance. In the freehand fluoroscopy-guided cohort (71 patients, 441 screws), screws were inserted using anatomical landmarks and lateral fluoroscopic guidance. Patients treated before 2009 were included in the fluoroscopy cohort, whereas those treated since mid-2009 (when the robot was acquired) were included in the robot cohort. Since then, the decision to operate using robotic assistance or conventional freehand technique has been based on surgeon preference and logistics. The accuracy of screw placement was assessed based on the Gertzbein-Robbins scale by a neuroradiologist blinded to treatment group. The radiological slice with the largest visible deviation from the pedicle was chosen for grading. A pedicle breach of $2 \mathrm{~mm}$ or less was deemed acceptable (Grades A and B) while deviations greater than $2 \mathrm{~mm}$ (Grades C, D, and E) were classified as misplacements.

RESULTS In the robot-assisted cohort, a perfect trajectory (Grade A) was observed for 366 screws (83.4\%). The remaining screws were Grades $B(n=44[10 \%]), C(n=15[3.4 \%]), D(n=8[1.8 \%])$, and $E(n=6[1.4 \%])$. In the fluoroscopy-guided group, a completely intrapedicular course graded as $A$ was found in $76 \%(n=335)$. The remaining screws were Grades $B(n=57[12.9 \%]), C(n=29[6.6 \%]), D(n=12[2.7 \%])$, and $E(n=8[1.8 \%])$. The proportion of nonmisplaced screws (corresponding to Gertzbein-Robbins Grades A and B) was higher in the robot-assisted group (93.4\%) than the freehand fluoroscopy group $(88.9 \%)(p=0.005)$.

CONCLUSIONS The authors' retrospective case review found that robot-guided pedicle screw placement is a safe, useful, and potentially more accurate alternative to the conventional freehand technique for the placement of thoracolumbar spinal instrumentation.
\end{abstract}

https://thejns.org/doi/abs/10.3171/2017.3.FOCUS179

KEY WORDS robotic surgery; spinal fusion; pedicle screw; spine instrumentation; computer-assisted surgery

$\mathrm{T}$ HE insertion of pedicle screws remains one of the crucial steps in thoracolumbar posterior instrumentation. Improvement of techniques that can facilitate procedures and increase accuracy in screw insertion is of crucial importance because the incorrect positioning of screws is associated with complications such as neurologi- cal deficits or vascular injuries. ${ }^{2}$ Pedicle screw insertion can be challenging, especially in patients with a spinal deformity such as scoliosis and in revision surgery where the anatomical landmarks have been altered.5,16 Many important structures, such as the spinal cord, nerve root, and vessels, are in close proximity to the pedicle. ${ }^{19}$ The reported 
rates of complications related to pedicle screw misplacement range from $1 \%$ up to $54 \%$. $3,16,19$

The robotic surgical assistance system SpineAssist (Mazor) was developed to increase the accuracy of pedicle screw trajectories. ${ }^{15,17}$ After preoperative imaging acquisition and planning of the desired screw trajectories, intraoperative registration is performed using 2 fluoroscopic images that are matched with the preoperative CT scan. The robot is mounted on a bridge that can be attached to the patient's spine, the iliac crest, or the operating table. The guidance arm moves to provide the surgeon with the preplanned trajectory. It is therefore a semi-active robotic system. The surgeon manually performs the drilling and screw insertion using an open or percutaneous technique.

The aim of this study was to compare the accuracy of robot-guided screw insertion with fluoroscopy-guided conventional technique in thoracolumbar surgery.

\section{Methods}

After institutional review board approval was obtained, we analyzed radiological data obtained in 169 patients who had undergone posterior instrumentation for spinal instability. Surgeries were performed by 2 senior surgeons (E.T. and B.S.) at 2 centers, from 2007 to 2015. This study is an update from our database of some patients reported on in 2014, with the addition of 74 new patients operated on at 2 reference centers. ${ }^{13}$ All patients had complete imaging studies including pre- and postoperative CT scans. Inclusion criteria were thoracolumbar posterior fusion to treat trauma, tumor, or degenerative disease. In the robot cohort (98 patients, 439 screws), open or percutaneous screw insertion was performed. In the fluoroscopy cohort (71 patients, 431 screws), screws were inserted freehand using anatomical landmarks and lateral fluoroscopic guidance. Patients operated on before 2009 were included in the fluoroscopy-assisted cohort, whereas those treated since mid-2009, the period when the Mazor robot was acquired, were included in the robot-guided cohort. From there, the decision to operate using robotic assistance or conventional freehand technique was based on surgeon's discretion and logistical reasons.

The primary outcome measure was the accuracy of screw placement evaluated on a postoperative CT scan according to the scale proposed by Gertzbein and Robbins (from Grade A to E: A, perfect intrapedicular localization; $\mathrm{B},<2$-mm pedicle breach; $\mathrm{C},<4-\mathrm{mm}$ pedicle breach; $\mathrm{D}$, $<6$-mm pedicle breach; and $\mathrm{E}, \mathrm{a} \geq 6$ - $\mathrm{mm}$ deviation from the pedicle cortex (Fig. 1). ${ }^{4}$ The radiological slice with the largest deviation from the pedicle was chosen for grading after analysis of axial, coronal, and sagittal views. A neuroradiologist who was blinded to the type of treatment evaluated all CT scans in both groups. Screws graded as $\mathrm{A}$ and $\mathrm{B}$ were considered to be clinically acceptable and were compared with the remaining "non-acceptable" screws using the Fisher chi-square test with a 2-sided significance level set at $<0.05$.

\section{Robot-Guided Surgical Technique}

In the robot-guided group, pedicle screws were placed using robotic guidance. Three steps are necessary: preoperative planning, intraoperative registration, and robot-
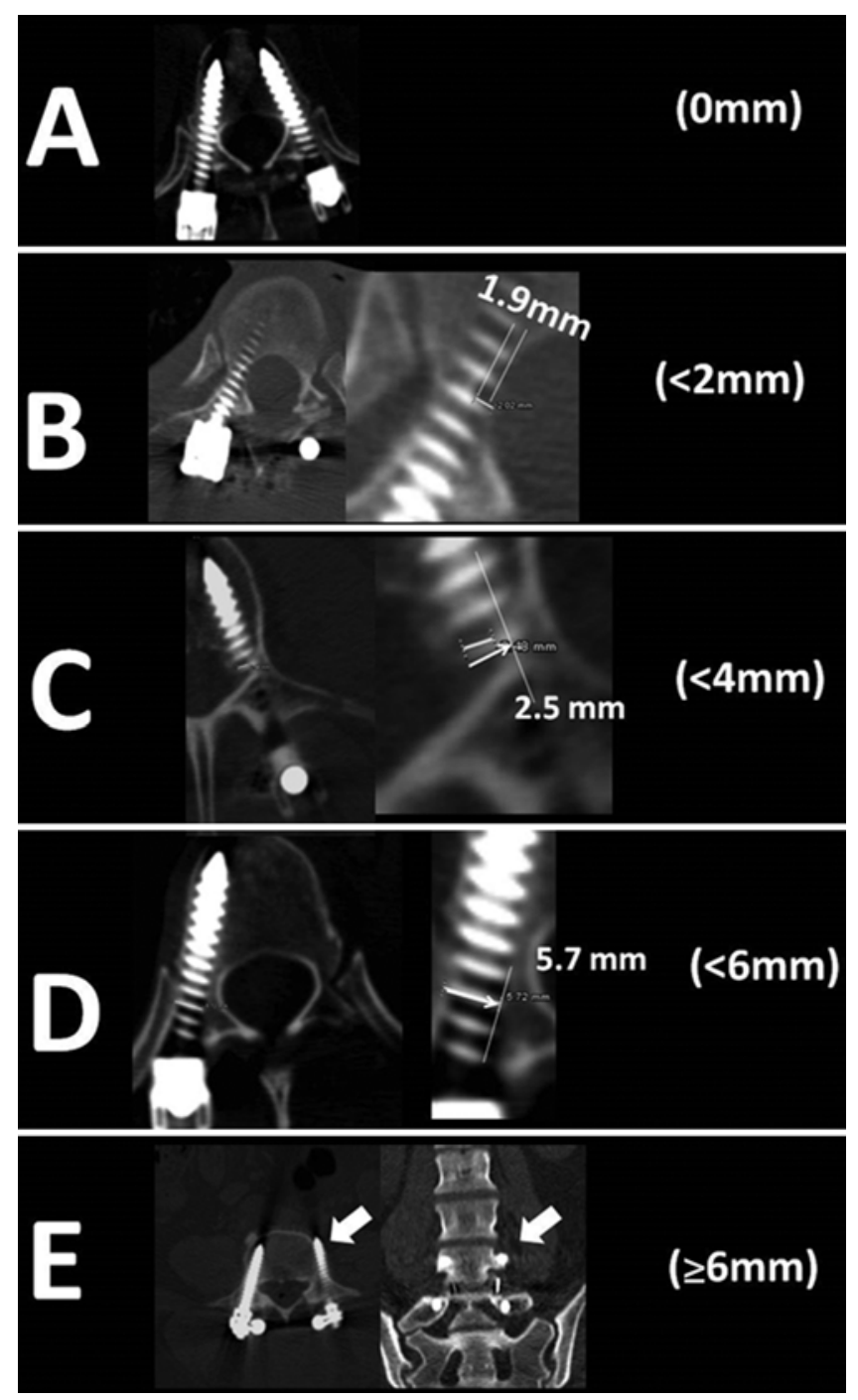

FIG. 1. CT scans demonstrating the Gertzbein-Robbins classification of screw positioning. The grades reflect the deviation of the screw from the "ideal" intrapedicular trajectory. Grade A is an intrapedicular screw without breach of the cortical layer of the pedicle; Grade B reflects a screw that breaches the cortical layer of the pedicle but does not exceed it laterally by $>2 \mathrm{~mm}$. Grades $\mathrm{C}$ and $\mathrm{D}$ indicate a penetration of $<4$ and $<6 \mathrm{~mm}$, respectively. Grade $\mathrm{E}$ indicates a screw that does not pass through the pedicle or that, at any given point in its intended intrapedicular course, breaches the cortical layer of the pedicle in any direction by $6 \mathrm{~mm}$ or more. Modified with permission from Schatlo et al: J Neurosurg Spine 20:636-243, 2014.

guided screw placement. The first step involves a preoperative analysis of the anatomy of the patient on the basis of a preoperative CT scan. Planning of screw insertion is carried out on the SpineAssist image-processing unit, including the determination of the screw entry point, the size of screws, and the trajectories planned in axial, coronal and sagittal views. Thus planning was done before the surgery on a computer, but it can also be done in the operating room on the robot console.

Intraoperatively, the patient is in the prone position and a clamp is rigidly fixed on the spinous process. This is followed by placement of 2 additional pins in the spinous 
TABLE 1. Baseline characteristics

\begin{tabular}{lcccc}
\hline \multicolumn{1}{c}{ Characteristic } & Overall & $\begin{array}{c}\text { Robot } \\
\text { Group }\end{array}$ & $\begin{array}{c}\text { Freehand } \\
\text { Group }\end{array}$ & $\begin{array}{c}p \\
\text { Value }\end{array}$ \\
\hline No. of patients* & 169 & 98 & 71 & \\
\hline Sex & & & & \\
\hline Female & 83 & 48 & 35 & 1.00 \\
\hline$\quad$ Male & 86 & 50 & 36 & 1.00 \\
\hline Age (yrs) $\dagger$ & $57.6 \pm 5$ & $58.3 \pm 12.8$ & $54.4 \pm 17$ & 0.33 \\
\hline Screws per case $\dagger$ & $5.21 \pm 3.23$ & $4.47 \pm 2.18$ & $6.21 \pm 2.87$ & 0.03 \\
\hline Pathology* & & & & \\
\hline Degenerative & 132 & $83(84.7)$ & $49(69)$ & 0.02 \\
\hline Tumor & 11 & $5(5.1)$ & $6(8.5)$ & \\
\hline Trauma & 26 & $10(10.2)$ & $16(22.5)$ & \\
\hline
\end{tabular}

* Values are the number or the number (\%) of patients.

$\dagger$ Values are the mean \pm SD.

process above and below. This bone mounting ensures that the robot is fixed to the patient's vertebrae and avoids errors attributed to inaccuracy in previous trials. ${ }^{11}$

To indicate the particular location of the mounting platform on fluoroscopy, a 3D marker is attached to it. Two fluoroscopic images of the 3D marker and spine are taken in anteroposterior and oblique views. The software automatically matches the intraoperative images to the corresponding locations on the preoperative CT.

To begin the surgical procedure, the surgeon selects the target vertebra from the preoperative plan, and the robot steers toward the chosen trajectory and indicates to the surgeon the entry point and direction of pedicle screw trajectory. The surgeon checks the suggested trajectory with a fluoroscopic image. After making a stab incision through skin and fascia, the surgeon inserts the cannula further until reaching the surface of the bone; drilling is performed, and screws are inserted through a K-wire. In our first 20 cases, drill advancement was followed on lateral fluoroscopy.

\section{Freehand Fluoroscopy-Guided Surgical Technique}

In the freehand group, the patient is placed in the prone position and the spine is prepared subperiostially to expose the transverse process, facet joint, and isthmus, which are used as anatomical landmarks for the entry point for screws. The pedicle is perforated with a probe and palpator. Screws are inserted using lateral fluoroscopic guidance and a K-wire. An anteroposterior fluoroscopic image is obtained at the end of this process to ensure that the screws are sufficiently convergent and correctly placed with respect to the medial border of the pedicle.

\section{Statistical Analysis}

Group comparisons were made using Fisher's exact with chi-square tests with a significance level of $\mathrm{p}<0.05$. Continuous variables were compared using the MannWhitney U-test. Binary multivariate regression was used to assess whether obesity (body mass index [BMI] $>30$ $\mathrm{kg} / \mathrm{m}^{2}$ ) or osteoporosis (bone density below 2.5 SDs below a reference population) affected the likelihood of a given
TABLE 2. Pedicle screw placement accuracy according to the Gertzbein and Robbins classification

\begin{tabular}{lccc}
\hline \multirow{2}{*}{$\begin{array}{l}\text { Screw } \\
\text { Grade }\end{array}$} & \multicolumn{2}{c}{ No. of Patients (\%) } & \multirow{2}{p}{} \\
\cline { 2 - 3 } & Robot Group & Freehand Group & Value \\
\hline A & $366(83.4)$ & $335(75)$ & \\
\hline B & $44(10)$ & $57(12.9)$ & \\
\hline C & $15(3.4)$ & $29(6.6)$ & \multirow{2}{*}{0.005} \\
\hline D & $8(1.8)$ & $12(2.7)$ & \\
\hline E & $6(1.4)$ & $8(1.8)$ & \\
\hline Total & $439(100)$ & $441(100)$ & \\
\hline
\end{tabular}

patient to have screw misplacement. SPSS 18.0 (IBM) was used to perform the statistical analyses.

\section{Results \\ Baseline Characteristics}

Most baseline characteristics did not differ between the robot-guided and freehand fluoroscopy-guided groups (Table 1). The distribution between men and women in both groups was homogeneous.

\section{Primary Outcome}

We analyzed the accuracy of pedicle screw fixation in 169 consecutives patients (83 women and 86 men) in whom lumbar thoracolumbar instrumentation was placed from 2010 to 2015 (Table 1). A detailed listing of pedicle screw accuracy grades is provided in Table 2. Overall, the analysis highlighted a clear improvement of accuracy for the robot cohort compared with the freehand cohort. In the robot group, a perfect trajectory (Gertzbein-Robbins Grade A) was observed for 366 (83.4\%) of the 439 screws. The remaining screws were Grades B $(n=44[10 \%]), C$ $(n=15[3.4 \%]), D(n=8[1.8 \%])$, and $E(n=6[1.4 \%])$. In the freehand fluoroscopy-guided group, a completely intrapedicular course of Grade A was found in $76 \%$ of the screws ( $\mathrm{n}=335$ of 441 screws). The remaining screws were Grades B $(n=57$ [12.9\%]), C $(n=29$ [6.6\%]), D ( $=12[2.7 \%])$, and $\mathrm{E}(\mathrm{n}=8[1.8 \%])$. The rate of "clinically acceptable" screw positions (which corresponds to Grade $\mathrm{A}$ and $\mathrm{B}$ ) was higher in the robot group than the freehand group, $93.4 \%$ and $88.9 \%$, respectively $(\mathrm{p}=0.005)$. The number of screws that exhibited a poor trajectory (Grades $\mathrm{C}, \mathrm{D}$, and E) was significantly lower in the robot group than the freehand group (29 [6.6\%] of 439 screws vs 49 [11.1\%] of 441 screws, respectively; $p=0.005$ ).

The rate of screw misplacement was lowest when using the robot in the lumbosacral region of the spine $(5.9 \%$ in the freehand group vs $2.1 \%$ in the robot group; $\mathrm{p}<0.001$ ) (Table 3). However, no difference was found with respect to thoracic screws $(\mathrm{p}=0.378)$.

Multivariate regression analysis did not confirm a potential role of either osteoporosis or BMI as predictors of screw misplacement in our cohort. In the freehand group, the odds ratio for screw misplacement in patients with BMI > $30 \mathrm{~kg} / \mathrm{m}^{2}$ was 1.7 (95\% CI 0.4-6.5; $\mathrm{p}=0.46$ ); the odds ratio for osteoporotic patients was 1.2 (95\% CI 0.3$4.4 ; p=0.78)$. In the robot group, the odds ratio for screw 
TABLE 3. Comparison of screw misplacement by spinal region

\begin{tabular}{|c|c|c|c|c|c|}
\hline \multirow[b]{2}{*}{$\begin{array}{l}\text { Spinal } \\
\text { Region }\end{array}$} & \multicolumn{2}{|c|}{ Robot Group (\%) } & \multicolumn{2}{|c|}{ Freehand Group (\%) } & \multirow[b]{2}{*}{$\begin{array}{c}p \\
\text { Value }\end{array}$} \\
\hline & $\begin{array}{c}\text { Total } \\
\text { No. of } \\
\text { Screws }\end{array}$ & $\begin{array}{c}\text { No. of } \\
\text { Misplaced } \\
\text { Screws }\end{array}$ & $\begin{array}{c}\text { Total } \\
\text { No. of } \\
\text { Screws }\end{array}$ & $\begin{array}{c}\text { No. of } \\
\text { Misplaced } \\
\text { Screws }\end{array}$ & \\
\hline Thoracic & 60 & $6(10)$ & 144 & $22(15.2)$ & 0.378 \\
\hline Lumbosacral & 379 & $9(2.3)$ & 297 & $26(8.8)$ & $<0.001$ \\
\hline Total & 439 & $15(3.4)$ & 441 & $48(10.9)$ & \\
\hline
\end{tabular}

misplacement in case of obesity was $1.6(95 \%$ CI $0.2-16.7$; $\mathrm{p}=0.71)$, whereas for osteoporosis it was $<0.1$ ( 0 -not applicable; $\mathrm{p}=0.99$ ).

\section{Discussion}

The effectiveness and the improvement of accuracy obtained by using the SpineAssist robotic system was the topic of this retrospective study, which included 169 patients. The analysis highlighted a clear improvement in accuracy when robotic guidance used to place screws was compared with the freehand fluoroscopic guidance. Effectively, in the robot group a perfect trajectory (Grade A) was observed for 366 screws $(83.4 \%)$ whereas the same grade was seen for 335 screws (76\%) in the freehand fluoroscopy group. Moreover, the rate of "clinically acceptable" screw positions (which correspond to A and B screws) was significantly higher in the robot group than the freehand group (93.4\% and $88.9 \%$, respectively). The total rate of misplaced thoracolumbar screws was lower in the robot group, but this difference was not statistically significant for the thoracic screws, which can be explained by a statistical bias generated by the fact that there are more screws in the freehand group at the thoracic level than in the robot group.

Improving accuracy and safety in pedicle screw insertion has led to the development of multiple new techniques such as image guidance with navigation, intraoperative imaging, and robot-assisted spine surgery. Of course, a learning curve is necessary for each new technique introduced into the market. It has been estimated that a minimum of 25 procedures is necessary for mastering the technique involved in using the first-generation Mazor robotic system. ${ }^{13}$ However, the more recent generation seems to be less demanding in terms of technique. ${ }^{9}$ For surgeons not acquainted with minimally invasive techniques, robotic surgery could facilitate its adoption. The reduction in radiation exposure is also a significant argument in favor of robotic technology in the light of rising awareness of the need to reduce radiation exposure for operating room personnel. ${ }^{12}$

A review of the literature shows screw placement rates close to those obtained in our study. A randomized controlled study published by Roser et al. showed that $99 \%$ of screws were satisfactorily placed with robotic assistance compared with $98 \%$ in the freehand group..$^{12}$ In a study by a different group of authors, up to $98.3 \%$ of screws were shown to be within $2 \mathrm{~mm}$ of the preoperatively planned location when the SpineAssist system was used..$^{10}$ In the same trend, a retrospective multicenter study, Devito et al. found a rate of $98 \%$ of clinically acceptable screw placements when using the SpineAssist robot. ${ }^{1}$ One year later, Kantelhardt et al. performed a retrospective comparison of robot-assisted and conventionally placed screws and reported accuracy rates of $94.5 \%$ and $91.4 \%$, respectively. More recently, van Dijk et al. reported $97.9 \%$ accuracy in their retrospective review of 112 patients who underwent minimally invasive spine surgery involving robotic guidance. ${ }^{18}$

In contrast, the study from Ringel and colleagues found the conventional freehand technique to have superior accuracy compared with the SpineAssist robot technique, with good placement seen for $93 \%$ and $85 \%$ of screws, respectively.1 The lesser accuracy was attributed to the use of a bed-mounted frame that is not rigidly fixed to the patients' spines. Therefore, the risk of movement of the robot arm relative to the patient may have induced a higher rate of screw misplacement. We implemented the use of spinous process fixation to avoid this inaccuracy.

Recently, the latest-generation Mazor robotic systems, the Renaissance, has been shown to be accurate and useful in thoracolumbar screw placement. For example, Hyun et al. obtained better accuracy in the robot group than the fluoroscopic open surgery group. ${ }^{6} \mathrm{Kim}$ et al. published a randomized controlled trial of robot-assisted versus freehand pedicle screw fixation in 78 patients ( 37 with robotassisted and 41 freehand surgery), and they concluded that robot-assisted pedicle screw placement was associated with fewer proximal facet joint violations and better screw trajectories, but the rate of intrapedicular accuracy was not different between the groups. ${ }^{8}$

\section{Limitations of the Technique}

This study clearly shows that the risk for pedicle screw malpositioning exists despite the use of robotic system guidance. One possible explanation of that is the so-called skiving phenomenon. ${ }^{5}$ The percutaneously inserted cannula can skive on a bony surface, such as a facet bony spur, with potential inaccuracy of the drilling procedure. Using a specific tool that is able to flatten the entry point before cannula introduction may solve this problem.

In the current and a previous study we have shown that the screw malpositioning rate was higher at the sacral level than at the lumbar level, especially in cases traditional open surgery. ${ }^{14}$ The large lever arm related to the robotic cannula (often needed for approaching the S-1 entry point) and, laterally, the soft-tissue pressure exerted on the cannula itself might explain the higher inaccuracy rate observed in sacral pedicle screw insertion. We suggest performing the percutaneous approach through a stab incision at the sacral level, even in cases of traditional open surgery. Another way is to ensure good soft-tissue retraction when inserting sacral screws, which require a more lateral entry point and a more medially directed trajectory. Finally, although the addition of the robot was helpful, it cannot replace the knowledge of the surgical anatomy held by the surgeon and the surgeon's ability to manage unexpected intraoperative events. The last solution is to rely on the anatomical reference and to opt for insertion of the screws according to the traditional technique of freehand surgery.

Installation of the robotic clamp on spinous processes 
and the use of the software during surgery increase the length of surgical procedure compared with the conventional freehand technique, especially in the surgeon's very first cases. Compared with intraoperative imaging and navigation setup, the robotic system adds little clutter to the conventional work space. The robot console takes up the space of an additional instrument table. In addition, the $\mathrm{C}$-arm needs to be equipped with a fiducial array. Whether the costs of acquisition and the expenses incurred by additional sterile material make up for hypothetical clinical benefit remains to be determined in future studies.

\section{Conclusions}

Our retrospective case review found that robot-guided pedicle screw placement is a safe, useful, and potentially more accurate alternative to conventional techniques for the placement of thoracolumbar spinal instrumentation.

\section{References}

1. Devito DP, Kaplan L, Dietl R, Pfeiffer M, Horne D, Silberstein B, et al: Clinical acceptance and accuracy assessment of spinal implants guided with SpineAssist surgical robot: retrospective study. Spine (Phila Pa 1976) 35:2109-2115, 2010 [Erratum in Spine (Phila Pa 1976) 36:91, 2011]

2. Gaines RW Jr: The use of pedicle-screw internal fixation for the operative treatment of spinal disorders. J Bone Joint Surg Am 82-A:1458-1476, 2000

3. Gautschi OP, Schatlo B, Schaller K, Tessitore E: Clinically relevant complications related to pedicle screw placement in thoracolumbar surgery and their management: a literature review of 35,630 pedicle screws. Neurosurg Focus 31(4):E8, 2011

4. Gertzbein SD, Robbins SE: Accuracy of pedicular screw placement in vivo. Spine (Phila Pa 1976) 15:11-14, 1990

5. Hu X, Ohnmeiss DD, Lieberman IH: Robotic-assisted pedicle screw placement: lessons learned from the first 102 patients. Eur Spine J 22:661-666, 2013

6. Hyun SJ, Kim KJ, Jahng TA, Kim HJ: Minimally invasive, robotic-vs. open fluoroscopic-guided spinal instrumented fusions - a randomized, controlled trial. Spine (Phila Pa 1976) [epub ahead of print], 2016

7. Kantelhardt SR, Martinez R, Baerwinkel S, Burger R, Giese A, Rohde V: Perioperative course and accuracy of screw positioning in conventional, open robotic-guided and percutaneous robotic-guided, pedicle screw placement. Eur Spine J 20:860-868, 2011

8. Kim HJ, Jung WI, Chang BS, Lee CK, Kang KT, Yeom JS: A prospective, randomized, controlled trial of robot-assisted vs freehand pedicle screw fixation in spine surgery. Int J Med Robot [epub ahead of print], 2016

9. Kim HJ, Lee SH, Chang BS, Lee CK, Lim TO, Hoo LP, et al: Monitoring the quality of robot-assisted pedicle screw fixation in the lumbar spine by using a cumulative summation test. Spine (Phila Pa 1976) 40:87-94, 2015

10. Pechlivanis I, Kiriyanthan G, Engelhardt M, Scholz M, Lücke S, Harders A, et al: Percutaneous placement of pedicle screws in the lumbar spine using a bone mounted miniature robotic system: first experiences and accuracy of screw placement. Spine (Phila Pa 1976) 34:392-398, 2009

11. Ringel F, Stüer C, Reinke A, Preuss A, Behr M, Auer F, et al:
Accuracy of robot-assisted placement of lumbar and sacral pedicle screws: a prospective randomized comparison to conventional freehand screw implantation. Spine (Phila Pa 1976) 37:E496-E501, 2012

12. Roser F, Tatagiba M, Maier G: Spinal robotics: current applications and future perspectives. Neurosurgery 72 (Suppl 1): $12-18,2013$

13. Schatlo B, Martinez R, Alaid A, von Eckardstein K, Akhavan-Sigari R, Hahn A, et al: Unskilled unawareness and the learning curve in robotic spine surgery. Acta Neurochir (Wien) 157:1819-1823, 2015

14. Schatlo B, Molliqaj G, Cuvinciuc V, Kotowski M, Schaller K, Tessitore E: Safety and accuracy of robot-assisted versus fluoroscopy-guided pedicle screw insertion for degenerative diseases of the lumbar spine: a matched cohort comparison. J Neurosurg Spine 20:636-643, 2014

15. Sukovich W, Brink-Danan S, Hardenbrook M: Miniature robotic guidance for pedicle screw placement in posterior spinal fusion: early clinical experience with the SpineAssist. Int J Med Robot 2:114-122, 2006

16. Tjardes T, Shafizadeh S, Rixen D, Paffrath T, Bouillon B, Steinhausen ES, et al: Image-guided spine surgery: state of the art and future directions. Eur Spine J 19:25-45, 2010

17. Togawa D, Kayanja MM, Reinhardt MK, Shoham M, Balter A, Friedlander A, et al: Bone-mounted miniature robotic guidance for pedicle screw and translaminar facet screw placement: part 2-Evaluation of system accuracy. Neurosurgery (2 Suppl 1) 60:ONS129-ONS139, 2007

18. van Dijk JD, van den Ende RPJ, Stramigioli S, Köchling M, Höss N: Clinical pedicle screw accuracy and deviation from planning in robot-guided spine surgery: robot-guided pedicle screw accuracy. Spine (Phila Pa 1976) 40:E986-E991, 2015

19. Wang H, Zhou Y, Liu J, Han J, Xiang L: Robot assisted navigated drilling for percutaneous pedicle screw placement: a preliminary animal study. Indian J Orthop 49:452-457, 2015

\section{Disclosures}

Dr. Tessitore is a consultant for Mazor Robotics.

\section{Author Contributions}

Conception and design: Molliqaj, Schatlo, Schaller, Tessitore. Acquisition of data: Molliqaj, Schatlo, Alaid, Solomiichuk, Tessitore. Analysis and interpretation of data: Molliqaj, Schatlo, Schaller, Tessitore. Drafting the article: Molliqaj, Schatlo, Tessitore. Critically revising the article: Molliqaj, Schatlo, Schaller, Tessitore. Reviewed submitted version of manuscript: all authors. Approved the final version of the manuscript on behalf of all authors: Molliqaj. Statistical analysis: Molliqaj, Schatlo, Tessitore. Administrative/technical/material support: Molliqaj, Schatlo, Schaller, Tessitore. Study supervision: Molliqaj, Schatlo, Schaller, Tessitore.

\section{Supplemental Information Videos}

Video Abstract. https://vimeo.com/212582499.

\section{Correspondence}

Granit Molliqaj, Service de Neurochirurgie, Faculté de Médecine, Hôpitaux Universitaires de Genève, 4 Rue Gabrielle Perret Gentil, Genève 1211, Switzerland. email: granitmolliqaj@gmail.com. 